\title{
Highly lymphatic metastatic pancreatic cancer cells possess stem cell-like properties
}

\author{
GUOPEI LUO $^{1 *}$, JIANG LONG ${ }^{1 *}$, XIAOBO CUI $^{2}$, ZHIWEN XIAO $^{1}$, ZUQIANG LIU $^{1}$, \\ SI SHI ${ }^{1}$, LIANG LIU ${ }^{1}, \mathrm{CHEN} \mathrm{LIU}^{1}$, JIN XU ${ }^{1}, \mathrm{MIN} \mathrm{LI}^{2}$ and XIANJUN YU ${ }^{1}$ \\ ${ }^{1}$ Department of Pancreatic and Hepatobiliary Surgery, Fudan University Shanghai Cancer Center; \\ Department of Oncology, Shanghai Medical College, Fudan University; Pancreatic Cancer Institute, \\ Fudan University, Shanghai 200032, P.R. China; ${ }^{2}$ The Vivian L. Smith Department of Neurosurgery, \\ The University of Texas Medical School at Houston, Houston, TX 77030, USA
}

Received November 25, 2012; Accepted December 21, 2012

DOI: $10.3892 /$ ijo.2013.1780

\begin{abstract}
Cancer stem cells are thought to be the origin of tumor metastasis. However, evidence of cancer stem cells as the source of lymphatic metastasis in pancreatic cancer is not clear. In this study, we examined the stem cell-like properties of the highly lymphatic metastatic pancreatic cancer cells BxPC-3-LN. Compared with the parental BxPC-3 cells, the BxPC-3-LN cells showed stem cell-like properties, including high lymphatic metastasis potential, self-renewal ability and chemoresistance. In addition, the BxPC-3-LN cells also expressed higher levels of sonic hedgehog and migrating cancer stem cell surface markers (CD133 and CXCR4) compared to the parental BxPC-3 cells. The growth of BxPC-3-LN cells was significantly inhibited by gemcitabine combined with the sonic hedgehog inhibitor cyclopamine. The BxPC-3-LN cells expressed lower levels of let-7, miR-34, miR-107, miR-125, miR-128, miR-130, miR-132 and miR-141 than the parental BxPC-3 cells detected by microRNA PCR array, which were reported to have close relation to stem cell factors. This study provides evidence that cancer stem cells are the major sources of pancreatic cancer lymphatic metas-
\end{abstract}

Correspondence to: Dr Xianjun Yu, Department of Pancreatic and Hepatobiliary Surgery, Fudan University Shanghai Cancer Center, Department of Oncology, Shanghai Medical College, Fudan University, Pancreatic Cancer Institute, Fudan University, 270 Dong'An Road, Xuhui, Shanghai 200032, P.R. China

E-mail: yuxianjun88@hotmail.com

Dr Min Li, The Vivian L. Smith Department of Neurosurgery, The University of Texas Medical School at Houston, 6431 Fannin Street, MSE R266, Houston, TX 77030, USA

E-mail:min.li@uth.tmc.edu

${ }^{*}$ Contributed equally

Key words: stem cell, lymphatic metastasis, sonic hedgehog, microRNA tasis, and microRNAs may regulate lymphatic metastasis in pancreatic cancer through modulating cancer stem cells.

\section{Introduction}

Pancreatic cancer is a devastating disease with a poor 5-year survival rate of $6 \%$ (1). Local recurrences and systematic metastasis are the major reasons for treatment failure, even after curative operation for early stage tumors (2-4). Lymphatic metastases are one of the common routes for tumor recurrences and metastases in pancreatic cancer and also significantly contribute to its poor prognosis (5-7). However, the underlying mechanism of lymphatic metastases in pancreatic cancer is not clear (8).

Cancer stem cells (CSCs) are a subpopulation of tumor cells that has the capacity to self-renew (by symmetric and asymmetric division), sustains the heterogeneous lineages of cancer cells and continually maintains tumorigenesis (9). A growing body of evidence has indicated the critical role of CSCs in many solid tumors (10-12). In addition to contributing to primary tumor formation, CSCs are also key players in the metastatic processes (12). However, the role of CSCs in pancreatic cancer lymphatic metastasis has not been well elucidated.

We have previously established a pancreatic cancer cell line BxPC-3-LN with aggressive features and highly lymphatic metastatic potential, which can serve as an ideal platform to study the mechanism of lymphatic metastasis in pancreatic cancer $(8,13)$. Given the critical function of CSCs in cancer metastasis, we investigated the stem cell-like properties of BxPC-3-LN cells to confirm the involvement of CSCs in lymphatic metastases of pancreatic cancer. We also examined the expression profile of microRNAs (miRNAs) involved in CSCs and cancer metastasis.

\section{Materials and methods}

Cell, tissue culture and animal model. The human pancreatic cancer cell line BxPC-3 was purchased from the American Type Culture Collection (Rockville, MD, USA). Cells were 
cultured at $37^{\circ} \mathrm{C}$ in a humidified atmosphere of $95 \%$ air and $5 \% \mathrm{CO}_{2}$. Eight-week-old immunodeficient male mice (BALB/c $\mathrm{nu} / \mathrm{nu}$ and NOD/SCID) were obtained from Shanghai SLAC Laboratory Animal Co. Ltd, Shanghai, China. All animal procedures were approved by the Institutional of Animal Care Committee, Fudan University, Shanghai, China.

Lymphatic metastatic potential. The BxPC-3-LN pancreatic cancer cells with highly lymphatic metastatic capability were previously established by consecutive in vivo selection processes (8). Eight-week-old BALB/c mice were orthotopically injected with $1 \times 10^{6} / \mathrm{ml}$ cells into the pancreas $(n=3)$. Mice were sacrificed at 6-week end-points to examine lymphatic involvement and metastatic lymph nodes were confirmed by pathological examination. Histological features of primary tumors were examined by hematoxylin and eosin staining.

Flow cytometry. Flow cytometry was used to detect the surface markers of CD133 (Miltenyi Biotec, Bergisch Gladbach, Germany) and CXCR4 (eBioscience, San Diego, CA, USA) by using Beckman Coulter FC500 (Miami, FL, USA). Cells were collected, fixed by resuspensing in $10 \mathrm{ml}$ of $70 \%$ ethanol for $30 \mathrm{~min}$ and washed in PBS. They were incubated in PBS solution containing CD133 and CXCR4 antibody at $4^{\circ} \mathrm{C}$ for $40 \mathrm{~min}$. Quantitative values are means $\pm \mathrm{SEM}$ from 3 independent experiments.

Self-renewal ability. Eight-week-old NOD/SCID mice were injected with 10,000 cells into the right flank $(n=3)$. The formation of primary tumors was examined every week. Mice were sacrificed at 4-week end-points to check tumor formation.

Sphere formation of tumor cells was performed as previously described with a slight modification (14). Cells were maintained in serum-free DMEM/Ham's F12 medium (Invitrogen, Carlsbad, CA) containing 1\% N2 supplement (Invitrogen), 2\% B27 supplement (Invitrogen), epidermal growth factor $(10 \mathrm{ng} / \mathrm{ml}$; PeproTech), basic fibroblast growth factor $(20 \mathrm{ng} / \mathrm{ml}$; PeproTech), heparin $(2 \mu \mathrm{g} / \mathrm{ml}$; Sigma-Aldrich, St. Louis, MO) and plated at a density of 1,000 cells/ml in 6-well ultra-low cluster plates (Corning Incorporated, Corning, NY). Sphere formation was observed under light microscope.

Clone formation ability was examined as previously described (15). In brief, 500 single cells were added to a 6 -well culture plate. Culture medium was changed every 3 days. At 2-week end-points, cells were stained with hematoxylin solution. The number of colonies was counted under a light microscope.

Immunofluorescence. Immunofluorescence was performed as previously described (16). In brief, on preincubation with normal blocking serum (diluted 1:20 in PBS) for $30 \mathrm{~min}$, cells were incubated for $1 \mathrm{~h}$ with sonic hedgehog (Shh) antibody (Epitomics, Burlingame, CA, USA) at 1:100 dilution. The cells were labeled with an anti-rabbit IgG-PE secondary antibody (Santa Cruz Biotechnology, Santa Cruz, CA, USA) at 1:200 dilution. DNA was counterstained with DAPI. Microscope was used to observe the expression of specific markers.
$R T-P C R$. Shh gene expression was tested by reversetranscription polymerase chain reaction (RT-PCR). The total RNA of cells was extracted using TRIzol isolation reagent (Invitrogen). The primers used in the PCR reactions were designed using information from the human genomic data base (forward primer 5'-CGGAGCGAGGAAGGGAAAG-3' and reverse primer 5'-TTGGGGATAAACTGCTTGTAGGC-3').

Drug cytotoxicity assay. Drug cytotoxicity assay was performed as previously described with a slight modification (8). In brief, the BxPC-3-LN cells were plated at a density of $10^{4}$ cells per well into 96-multiwell plates and were allowed to grow for $24 \mathrm{~h}$. Cyclopamine (6 $\mu \mathrm{mol} / 1$, Sigma-Aldrich), gemcitabine (100 nM, Eli Lilly) and cyclopamine ( $6 \mu \mathrm{mol} / \mathrm{l})$ combined with gemcitabine $(100 \mathrm{nM})$ were added to the cells and then incubated for $48 \mathrm{~h}$ at $37^{\circ} \mathrm{C}$. After initial incubation, cells were incubated at $37^{\circ} \mathrm{C}$ for $4 \mathrm{~h}$ with $20 \mu \mathrm{l}$ of MTS (Promega) and absorbance was read at $492 \mathrm{~nm}$. Viability levels are presented as a percentage of the level obtained from the blank control (untreated cells) (mean $\pm S E, n=6$ ).

MicroRNA PCR array. Isolation of miRNAs from cells was carried out using the mirVana miRNA Isolation Kit (Applied Biosystems). miRNAs expression was quantified by using TaqMan miRNA qRT-PCR assays as previously described (TaqMan Array Human MicroRNA A+B Cards Set v3.0, Applied Biosystems) (17). The PCR reaction was performed on 7900HT Real-Time PCR System (Applied Biosystems) with the following conditions: $16^{\circ} \mathrm{C} 2 \mathrm{~min}, 42^{\circ} \mathrm{C} 1 \mathrm{~min}, 50^{\circ} \mathrm{C}$ $1 \mathrm{sec}$ for 40 cycles. The fold change of miRNAs expression (BxPC-3 vs. BxPC-3-LN) was calculated.

Statistical analysis. Fisher's exact test and Student's t-test were applied to compare enumeration data and measure mean $\mathrm{t}$ data. Stata 10.0 was used for the tests and $\mathrm{p}<0.05$ was considered statistically significant.

\section{Results}

Lymphatic metastasis potential. After 6 weeks of tumor cell implantation orthotopically, the BxPC-3-LN cells generated celiac axis, para-aortic and para-renal lymph node metastases confirmed by pathological examination, with larger primary tumors compared with the parental BxPC-3 cells (Fig. 1A and B). The distribution of metastatic lymph nodes by BxPC-3-LN cells was similar to human disease. No obvious lymphatic metastasis was observed for the parental BxPC-3 cells (Fig. 1A).

BXPC-3-LN cells possess CSC markers. In order to determine whether the lymphatic metastatic BxPC-3-LN cells have CSC-like properties, we examined the expression of the CSC markers $\mathrm{CD}_{133^{+}}$and $\mathrm{CXCR} 4^{+}$by flow cytometry. As shown in Fig. 2, the BxPC-3-LN cells contained an average of $1.0 \%$ of $\mathrm{CD}_{133}{ }^{+} / \mathrm{CXCR} 4^{+}$cells, which was 5 times of that in the BxPC-3 cells $(0.2 \%)$, indicating BxPC-3-LN cells possess CSC-like properties, which may explain the matastatic potential of this cell line.

Self-renewal ability. The self-renewal ability of the BxPC-3-LN and BxPC-3 cells was detected by in vivo 

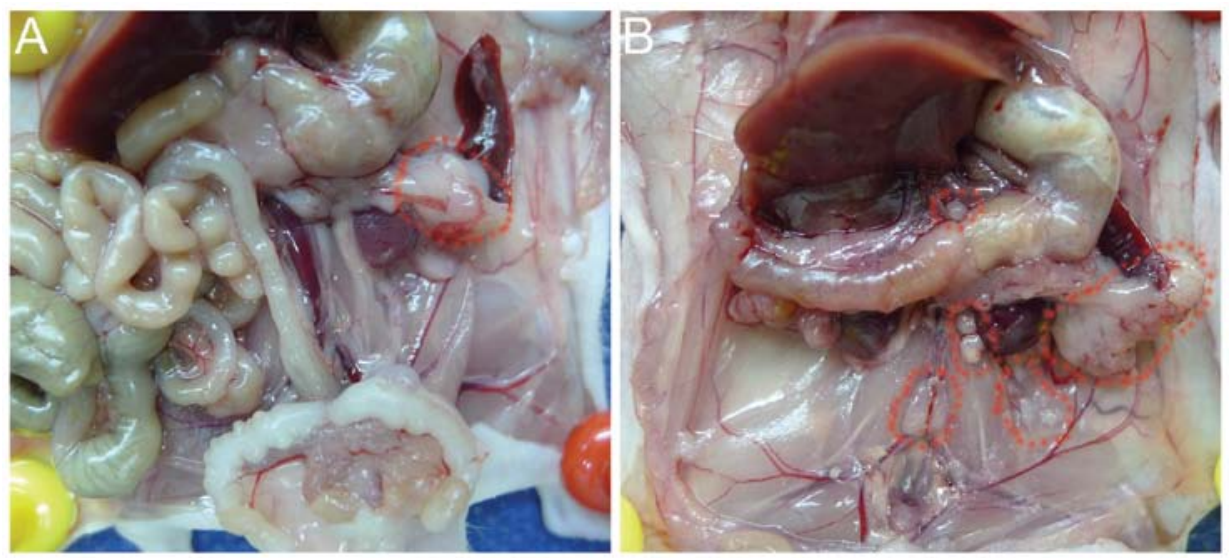

Figure 1. Lymphatic metastatic potential of the BxPC-3-LN and BxPC-3 cells. After 6 weeks of tumor cell orthotopic implantation, the BxPC-3-LN cells generated celiac axis, para-aortic and para-renal lymph node metastases, with large primary tumors compared with the parental BxPC-3 cells (A and B). (A) No obvious metastatic lymphatic metastasis was observed for the parental BxPC-3 cells.
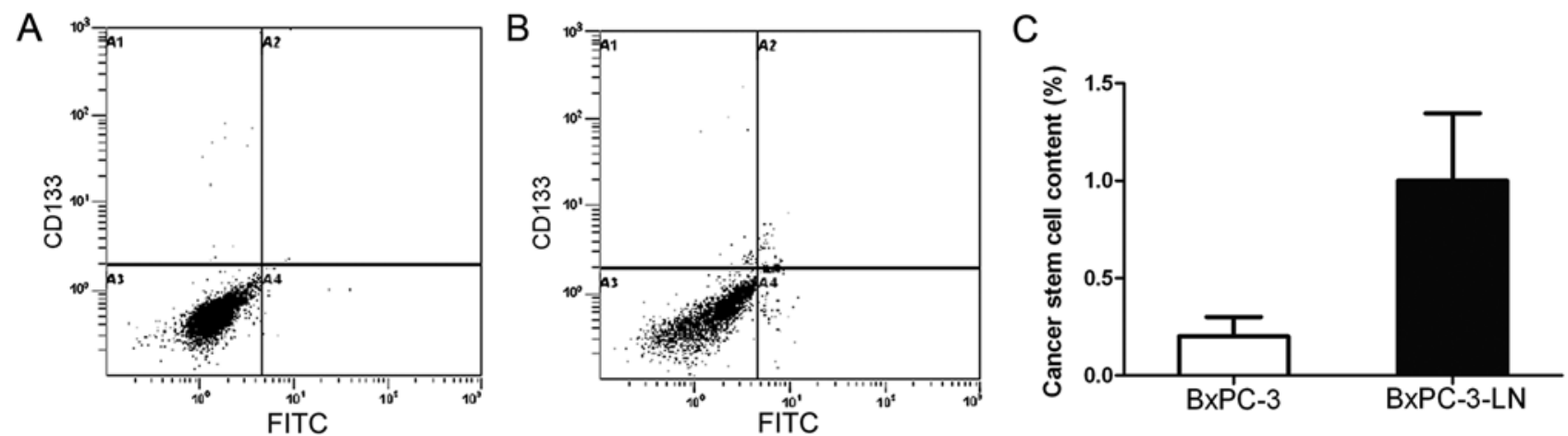

Figure 2. Expression of CSC markers examined by flow cytometry. The BxPC-3-LN cells contained an average of $1.0 \%$ of CD133 ${ }^{+} \mathrm{CXCR} 4^{+}$cells, which was 5 times that of BxPC-3 cells $(0.2 \%)$. Quantitative values are means \pm SEM from 3 independent experiments.
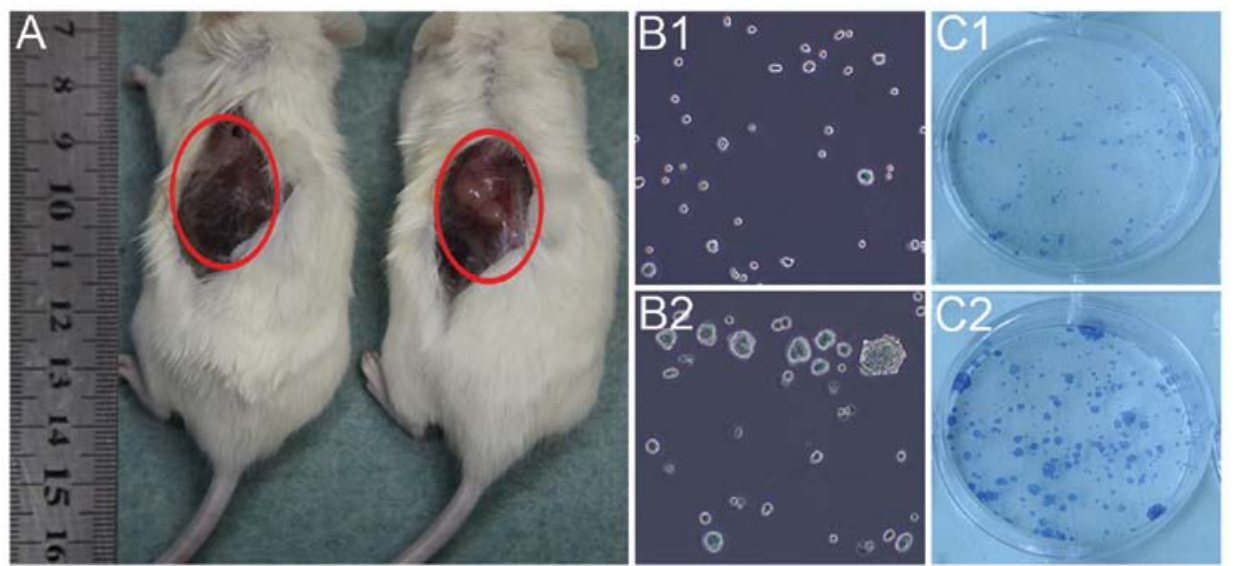

Figure 3. Self-renewal ability of the BxPC-3-LN and BxPC-3 cells. (A) A representative experiment depicted tumor formation in NOD-SCID mice at the injection site of 10,000 BxPC-3-LN cells (right), with no tumor formation seen at the injection site of 10,000 BxPC-3 cells (left). (B2) The BxPC-3-LN cells formed more spheres than the (B1) BxPC-3 cells at $72 \mathrm{~h}$ after plating (x100). (C2) The BxPC-3-LN cells formed increased clones compared with the (C1) BxPC-3 cells after 2 weeks of plating $(\mathrm{x} 40)$.

tumorigenicity and in vitro sphere and clone formation. The BxPC-3-LN cells generated big tumors with 10,000 cells injected after 4 week of implantation (Fig. 3A right), while no tumor was observed for BxPC-3 cells with the same amount 
A

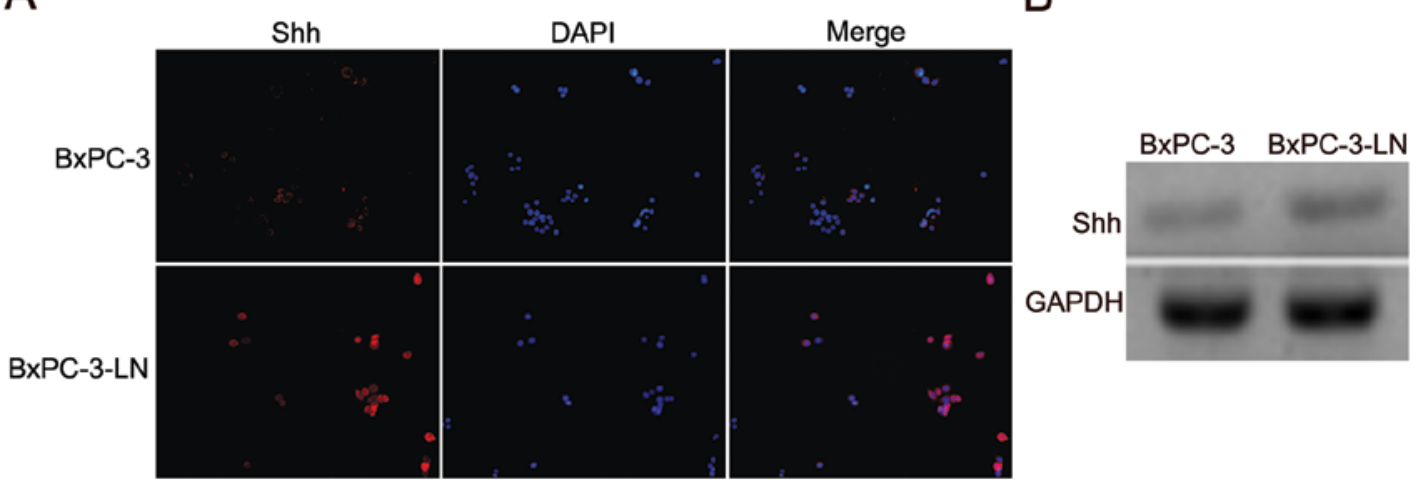

Figure 4. Shh expression by immunofluorescence and RT-PCR. The BxPC-3-LN cells showed high expression of Shh compared with the BxPC-3 cells by immunofluorescence (A, x200). (B) The upregulation of Shh in the BxPC-3-LN cells was also confirmed by RT-PCR.
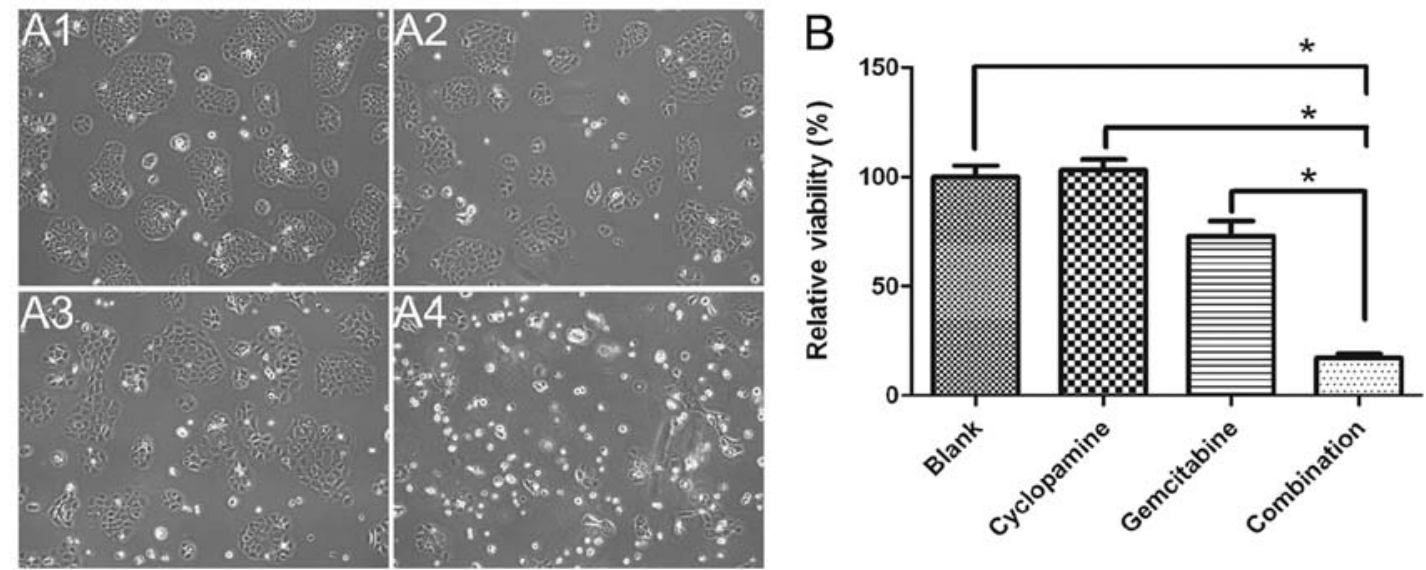

Figure 5. Inhibition effect of (A2) cyclopamine, (A3) gemcitabine and (A4) cyclopamine combined with gemcitabine on the growth of (A1) BxPC-3-LN cells compared with blank under a light microscope (x200). (B) Relative viability (\%) following $48 \mathrm{~h}$ gemcitabine, cyclopamine and cyclopamine combined with gemcitabine treatment was estimated by MTS assay. Viability levels are presented as a percentage of the level obtained from the blank control (untreated cells) $\left(\right.$ mean $\left.\pm \mathrm{SE}, \mathrm{n}=6,{ }^{*} \mathrm{p}<0.0001\right)$.

of cells injected (Fig. 3A left). In addition, the BxPC-3-LN cells showed more spheres (Fig. 3B2) and clones formation (Fig. 3C2) than the BxPC-3 cells (Fig. 3B1 and C1).

Shh expression is increased in BxPC-3-LN cells. The expression of Shh in BxPC-3 and the lymphatic metastatic BxPC-3-LN cells was examined by immunofluorescence. The BxPC-3-LN cells showed higher expression of Shh compared with the parental BxPC-3 cells (Fig. 4A). The upregulation of Shh mRNA in the BxPC-3-LN cells was also confirmed by RT-PCR (Fig. 4B). Those data suggest that Shh pathway might be involved in the lymphatic metastasis of pancreatic cancer.

Drug cytotoxicity. MTS assay was used to examine the growth inhibition of cyclopamine, gemcitabine and cyclopamine combined with gemcitabine in BxPC-3 cells. As shown in Fig. 5, cyclopamine combined with gemcitabine $(17.3 \%)$ caused significant inhibition of cell growth compared with gemcitabine alone $(\mathrm{p}<0.0001)$ and cyclopamine alone (p<0.0001) (Fig. 5), indicating that blocking Shh pathway is needed in controling the lymphatic metastasis of pancreatic cancer.

MicroRNA PCR array. Differential expression of miRNAs in the BxPC-3 and BxPC-3-LN cells was quantified by using TaqMan miRNA qRT-PCR assay. Compared with the BxPC-3 cells, several aberrantly expressed miRNAs related to CSCs were found in the BxPC-3-LN cells, including upregulation of miR-572, miR-206, miR-449a, miR-489, miR 184 and downregulation of let- $7 \mathrm{~g}-3 \mathrm{p}$, let7i-3p, let-7a-3p, miR-107, miR-128 and miR-141-5p (Fig. 6), suggesting a possible regulatory role of microRNA in lymphatic metastasis of pancreatic cancer.

\section{Discussion}

CSC is thought as the major source of tumorigenesis and metastasis (9). CD133 is a stem-like cell surface marker expressed in diverse solid tumors, including pancreatic, brain 


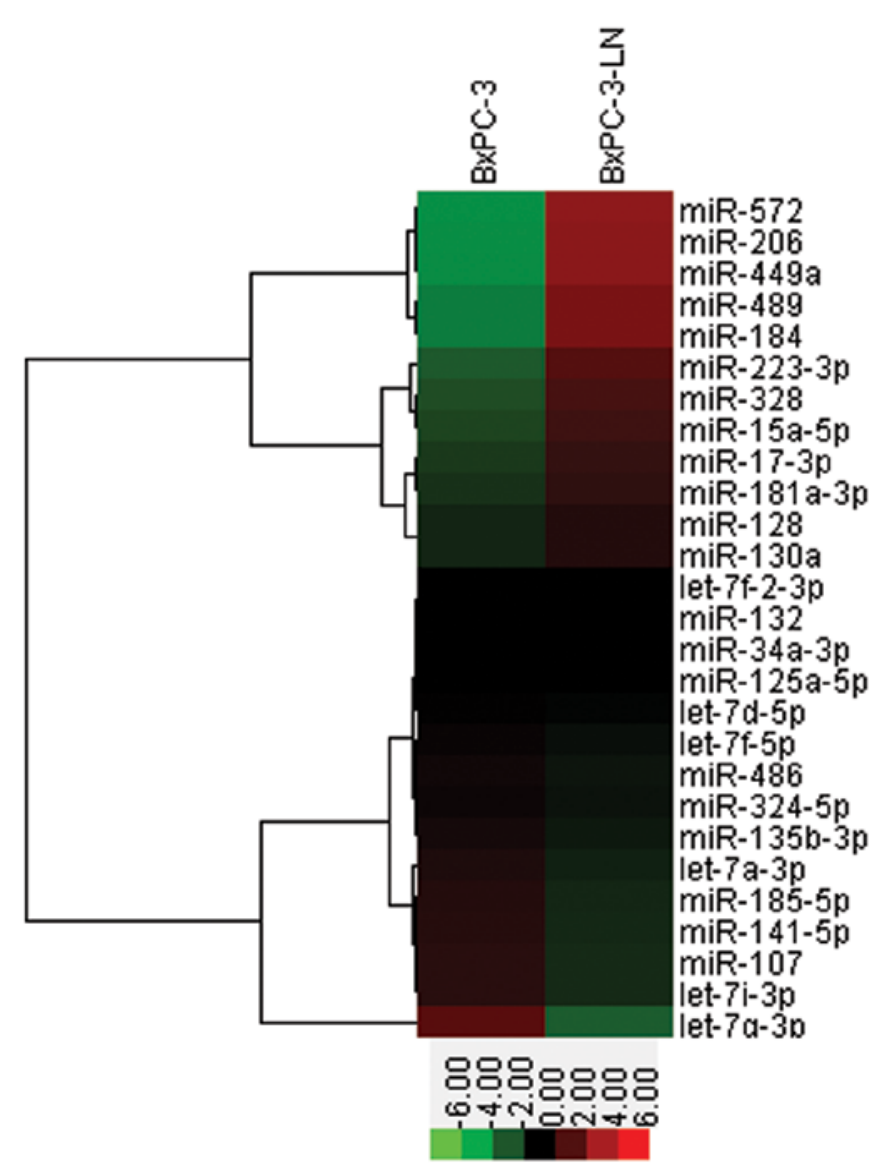

Figure 6. Aberrant expression of microRNAs related to cancer stem cells in the BxPC-3-LN cells compared with the parental BxPC-3 cells by microRNA PCR array.

and colon tumors $(12,18,19)$. In pancreatic cancer, CD133+ expressing cells were associated with tumor initiation, chemoresistance, increased cell migration and invasion $(12,20)$. More importantly, it was demonstrated that a $\mathrm{CD} 133^{+} / \mathrm{CXCR}^{+}$ subpopulation was responsible for pancreatic cancer metastasis (12). Previously, we have confirmed the migration, invasive and chemoresistant capability of a lymphatic metastatic pancreatic cancer cell line BxPC-3-LN established by serial in vivo selection (8). In this study, we further demonstrated that BxPC-3-LN cells presented stem cell-like properties, including highly lymphatic metastastic potential, self-renewal ability and chemoresistance. In addition, the BxPC-3-LN cells also expressed higher levels of Shh, which was considered as one of the most important stem cell-related signal pathways $(11,12)$. CD133 ${ }^{+} / \mathrm{CXCR}^{+}$cells, which were identified as the migrating CSCs in a previous study (12), were found to be enriched in the BxPC-3-LN cells compared with the parental BxPC-3 cells. Cyclopamine (a Shh signal pathway inhibitor) combined with gemcitabine showed greater inhibitory effect on the BxPC-3-LN cells than cyclopamine and gemcitabine alone. Our findings suggest that $\mathrm{CD} 133^{+} / \mathrm{CXCR}^{+}$ cells might be the major migrating CSCs and are responsible for the lymphatic metastases of pancreatic cancer.

Previous studies reported that gemcitabine-resistant cells showed cancer stem-like cell phenotype, which underwent epithelial-to-mesenchymal transition (EMT) and showed increased expression of the stem cell markers CD24, CD44 and epithelial specific antigen (ESA) $(21,22)$. These findings indicate that cancer stem-like cells, which are known for their chemoresistant ability, can be enriched during the acquisition of chemoresistance and in therapeutic treatments $(23,24)$. The in vivo serial selection process that was used shares common features to gemcitabine-resistant cells established by exposure to serially escalated doses of gemcitabine, which was also accompanied by an enrichment of CSCs.

miRNAs are a group of short non-coding RNAs that modulate gene expression at the post-transcriptional level, usually leading to translational suppression or gene silencing (25). miRNAs are involved in tumor maintenance, progression, and treatment resistance (26-28). There are also evidence showing that miRNAs regulate CSCs by suppressing the expression of 'stem cell factors' such as Sox2, Kif4, CD44 and Notch (24,26-32). Yu et al (24) found that the CSC-enriched cells expressed low levels of let-7, miR-107, miR-125, miR-128, miR-130, miR-132 and miR-141 compared with the parental cells. Another study showed that restoration of miR-34 strongly suppressed the growth and invasion of p53-mutant pancreatic cancer cells, and sensitized the cells to chemo- and radiotherapy. Moreover, restoration of miR-34 resulted in an $87 \%$ decrease in the $\mathrm{CD} 133^{+} / \mathrm{CXCR}^{+} \mathrm{CSC}$ by direct regulating its downstream targets Notch $1 / 2$ and Bcl-2, suggesting the potential role of miR-34 in CSCs (29). In our study, we also showed that the CSC-enriched BxPC-3-LN cells expressed lower levels of let-7, miR-34, miR-107, miR-125, miR-128, miR-130, miR-132 and miR-141 than the parental BxPC-3 cells, indicating miRNAs regulates the lymphatic metastasis of pancreatic cancer by regulating CSCs. In addition, we found upregulation of miR-184 in BxPC-3-LN cells, which is epigenetically regulated by Methyl-CpG binding protein 1 (MBD1) for modulating stem cell growth and differentiation (33). Our previous studies have demonstrated that MBD1 plays an important role in pancreatic cancer growth and lymphatic metastasis $(34,35)$. These findings suggest that MBD1 regulate pancreatic cancer progression by epigenetic regulation of miR-184, which can affect stem cell properties.

In conclusion, the BxPC-3-LN cells possess stem cell-like properties. The in vivo serial selection processes were similar to gemcitabine-resistant cells established by exposure to serially escalated doses of gemcitabine, which was also accompanied by an enrichment of CSCs. Our results suggest the function of CSCs in pancreatic cancer lymphatic metastasis and miRNAs may regulate pancreatic cancer metastasis through modulating the properties of CSCs.

\section{Acknowledgements}

This study was supported in part by the National Science Foundation of China (grant nos. 81172276, 81101807, 81001058, 30901435 and 30972905) (X.Y.), the Shanghai Science and Technology Commission (grant no. 12QH1400600), '985 project' Third Stage Oncology Project (grant no. 985III-YFX0102) (J.L.), the MacDonald Research Fund, and the William and Ella Owens Medical Research Foundation (M.L.). We thank Dr Bo Zhang and Dr Huanyu Xia for their technical assistance. 


\section{References}

1. Jemal A, Siegel R, Ward E, Hao Y, Xu J and Thun MJ: Cancer statistics, 2009. CA Cancer J Clin 59: 225-249, 2009.

2. Luo G, Long J, Zhang B, et al: Stroma and pancreatic ductal adenocarcinoma: An interaction loop. Biochim Biophys Acta 1826: $170-178,2012$

3. Warshaw AL and Fernandez-del Castillo C: Pancreatic carcinoma. N Engl J Med 326: 455-465, 1992.

4. Li X, Ma Q, Xu Q, et al: SDF-1/CXCR4 signaling induces pancreatic cancer cell invasion and epithelial-mesenchymal transition in vitro through non-canonical activation of Hedgehog pathway. Cancer Lett 322: 169-176, 2012.

5. Pedrazzoli S, DiCarlo V, Dionigi R, et al: Standard versus extended lymphadenectomy associated with pancreatoduodenectomy in the surgical treatment of adenocarcinoma of the head of the pancreas: a multicenter, prospective, randomized study. Lymphadenectomy Study Group. Ann Surg 228: 508-517, 1998.

6. Li Y, Kong D, Ahmad A, Bao B and Sarkar FH: Pancreatic cancer stem cells: Emerging target for designing novel therapy. Cancer Lett: May 20, 2012 (Epub ahead of print).

7. Ni X, Yang $J$ and Li M: Imaging-guided curative surgical resection of pancreatic cancer in a xenograft mouse model Cancer Lett 324: 179-185, 2012.

8. Long J, Luo G, Liu C, et al: Development of a unique mouse model for pancreatic cancer lymphatic metastasis. Int J Oncol 41: 1662-1668, 2012.

9. Clarke MF, Dick JE, Dirks PB, et al: Cancer stem cells - perspectives on current status and future directions: AACR Workshop on cancer stem cells. Cancer Res 66: 9339-9344, 2006.

10. Visvader JE and Lindeman GJ: Cancer stem cells in solid tumours: accumulating evidence and unresolved questions. Nat Rev Cancer 8: 755-768, 2008.

11. Li C, Heidt DG, Dalerba P, et al: Identification of pancreatic cancer stem cells. Cancer Res 67: 1030-1037, 2007.

12. Hermann PC, Huber SL, Herrler T, et al: Distinct populations of cancer stem cells determine tumor growth and metastatic activity in human pancreatic cancer. Cell Stem Cell 1: 313-323, 2007.

13. Luo G, Yu X, Jin C, et al: LyP-1-conjugated nanoparticles for targeting drug delivery to lymphatic metastatic tumors. Int J Pharm 385: 150-156, 2010.

14. Singh SK, Clarke ID, Terasaki M, et al: Identification of a cancer stem cell in human brain tumors. Cancer Res 63: 5821-5828, 2003.

15. Shi WD, Meng ZQ, Chen Z, Lin JH, Zhou ZH and Liu LM Identification of liver metastasis-related genes in a novel human pancreatic carcinoma cell model by microarray analysis. Cancer Lett 283: 84-91, 2009.

16. Giodini A, Kallio MJ, Wall NR, et al: Regulation of microtubule stability and mitotic progression by survivin. Cancer Res 62: 2462-2467, 2002

17. Mitchell PS, Parkin RK, Kroh EM, et al: Circulating microRNAs as stable blood-based markers for cancer detection. Proc Natl Acad Sci USA 105: 10513-10518, 2008.
18. O'Brien CA, Pollett A, Gallinger S and Dick JE: A human colon cancer cell capable of initiating tumour growth in immunodeficient mice. Nature 445: 106-110, 2007.

19. Singh SK, Hawkins C, Clarke ID, et al: Identification of human brain tumour initiating cells. Nature 432: 396-401, 2004.

20. Moriyama T, Ohuchida K, Mizumoto K, et al: Enhanced cell migration and invasion of $\mathrm{CD}_{133^{+}}$pancreatic cancer cells cocultured with pancreatic stromal cells. Cancer 116: 3357-3368, 2010

21. Shah AN, Summy JM, Zhang J, Park SI, Parikh NU and Gallick GE: Development and characterization of gemcitabineresistant pancreatic tumor cells. Ann Surg Oncol 14: 3629-3637, 2007.

22. Wang Z, Li Y, Kong D, et al: Acquisition of epithelialmesenchymal transition phenotype of gemcitabine-resistant pancreatic cancer cells is linked with activation of the notch signaling pathway. Cancer Res 69: 2400-2407, 2009.

23. Hong SP, Wen J, Bang S, Park S and Song SY: CD44-positive cells are responsible for gemcitabine resistance in pancreatic cancer cells. Int J Cancer 125: 2323-2331, 2009.

24. Yu F, Yao $\mathrm{H}$, Zhu $\mathrm{P}$, et al: let-7 regulates self renewal and tumorigenicity of breast cancer cells. Cell 131: 1109-1123, 2007.

25. Hatfield SD, Shcherbata HR, Fischer KA, Nakahara K, Carthew RW and Ruohola-Baker H: Stem cell division is regulated by the microRNA pathway. Nature 435: 974-978, 2005.

26. Wellner U, Schubert J, Burk UC, et al: The EMT-activator ZEB1 promotes tumorigenicity by repressing stemness-inhibiting microRNAs. Nat Cell Biol 11: 1487-1495, 2009.

27. Liu C, Kelnar K, Liu B, et al: The microRNA miR-34a inhibits prostate cancer stem cells and metastasis by directly repressing CD44. Nat Med 17: 211-215, 2011.

28. Cheng H, Shi S, Cai X, et al: microRNA signature for human pancreatic cancer invasion and metastasis. Exp Ther Med 4: $181-187,2012$.

29. Ji Q, Hao X, Zhang M, et al: MicroRNA miR-34 inhibits human pancreatic cancer tumor-initiating cells. PLoS One 4: e6816, 2009.

30. Leal JA and Lleonart ME: MicroRNAs and cancer stem cells: Therapeutic approaches and future perspectives. Cancer Lett: Apr 30, 2012 (Epub ahead of print).

31. Ni X, Long J, Cen P, Chen L, Yang J and Li M: Pancreatic cancer tumour initiating cells: the molecular regulation and therapeutic values. J Cell Mol Med 16: 988-994, 2012.

32. Liu C and Tang DG: MicroRNA regulation of cancer stem cells Cancer Res 71: 5950-5954, 2011.

33. Liu C, Teng ZQ, Santistevan NJ, et al: Epigenetic regulation of miR-184 by MBD1 governs neural stem cell proliferation and differentiation. Cell Stem Cell 6: 433-444, 2010.

34. Luo G, Jin C, Long J, et al: RNA interference of MBD1 in BxPC-3 human pancreatic cancer cells delivered by PLGA-poloxamer nanoparticles. Cancer Biol Ther 8: 594-598, 2009.

35. Liu C, Chen Y, Yu X, et al: Proteomic analysis of differential proteins in pancreatic carcinomas: Effects of MBD1 knock-down by stable RNA interference. BMC Cancer 8: 121, 2008. 